On the 'fake' inferred entanglement associated with the maximum entropy inference of quantum states

To cite this article: J Batle et al 2001 J. Phys. A: Math. Gen. 346443

View the article online for updates and enhancements.
You may also like

- Estimating probability density functions
$\frac{\text { using a combined maximum entropy }}{\text { moments and Bayesian method. Theory }}$
$\frac{\text { and numerical examples }}{\text { N Armstrong, G J Sutton and D B Hibbert }}$
- $\frac{\text { Prediction of potential fishing zones for }}{\text { yellowfin tuna (Thunnus albacares) using }}$
maxent models in Aceh province waters
E S Y Siregar, V P Siregar, R Jhonnerie et
al.
- Data-driven quantification of the
robustness and sensitivity of cell signaling
$\frac{\text { networks }}{\text { Sayak Mukherjee, Sang-Cheol Seok, }}$
Veronica J Vieland et al.

Recent citations

- Inference schemes and entanglement determination

A. R. Plastino et al 


\title{
On the 'fake' inferred entanglement associated with the maximum entropy inference of quantum states
}

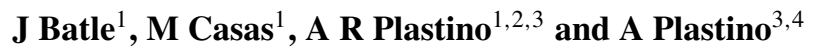 \\ ${ }^{1}$ Departament de Física, Universitat de les Illes Balears, 07071 Palma de Mallorca, Spain \\ 2 Faculty of Astronomy and Geophysics, National University La Plata, CC 727, 1900 La Plata, \\ Argentina \\ ${ }^{3}$ National Research Council (CONICET), Argentina \\ ${ }^{4}$ Department of Physics, National University La Plata, CC 727, 1900 La Plata, Argentina
}

Received 18 December 2000, in final form 17 May 2001

Published 10 August 2001

Online at stacks.iop.org/JPhysA/34/6443

\begin{abstract}
The inference of entangled quantum states by recourse to the maximum entropy (MaxEnt) principle is considered in connection with the recently pointed out problem of fake inferred entanglement (Horodecki R et al 1999 Phys. Rev. A 59 1799). We show that there are operators $\hat{A}$, both diagonal and non-diagonal in the Bell basis, such that, when the expectation value $\langle\hat{A}\rangle$ is taken as prior information, the problem of fake entanglement is not solved by adding a new constraint associated with the mean value of $\hat{A}^{2}$ (unlike what happens when the partial information is given by the expectation value of a Bell operator). The fake entanglement generated by the MaxEnt principle is also studied quantitatively by comparing the entanglement of formation of the inferred state with that of the original one.
\end{abstract}

PACS numbers: 03.67.-a, 89.70.+c, 03.65.Ta

\section{Introduction}

The inference of entangled quantum states by recourse to the maximum entropy (MaxEnt) principle has been recently considered in the literature [1-5]. In particular, the question of how to estimate in a reliable way the amount of entanglement of a bipartite quantum system when only partial, incomplete information about its state is available was addressed by Horodecki et al [1]. Various strategies have been advanced in order to tackle this problem [1,3-6]. Horodecki's question has also been considered in connection with procedures for the entanglement purification of unknown quantum states [7]. The motivation behind these lines of inquiry is that quantum entanglement is the basic resource required to implement several of the most important processes studied by quantum information theory [8-10], such as quantum cryptographic key distribution [11], quantum teleportation [12], superdense coding [13], and quantum computation $[14,15]$. A state of a composite quantum system is 
called 'entangled' if it cannot be represented as a mixture of factorizable pure states. Otherwise, the state is called separable. The above definition is physically meaningful because entangled states (unlike separable states) cannot be prepared locally by acting on each subsystem individually [16]. Nowadays there is general consensus on the fact that the phenomenon of entanglement is one of the most fundamental and non-classical features exhibited by quantum systems [8].

If one has enough information it is possible to determine the amount of entanglement of a quantum system even if the available information does not allow for a complete knowledge of the system's state. An interesting example of this situation was recently discussed by Sancho and Huelga [6], who studied the minimal experimental protocol required for determining the entanglement of a two-qubits pure state from local measurements. Another important result obtained by Sancho and Huelga is that the knowledge of the expectation value of just one observable (local or not) does not suffice to determine the entanglement of a given unknown pure state of two particles [6]. The case in which the prior information is not sufficient for a complete determination of the amount of entanglement was further examined by Horodecki et al [1]. These authors did not restrict their analysis to pure states. They assumed that the available information consists of the mean values of a given set of observables $\hat{A}_{i}$. Jaynes' MaxEnt principle $[17,18]$ provides a general inference scheme to treat this kind of situation. According to Jaynes' principle, one must choose the state yielding the least unbiased description of the system compatible with the available data. That state is provided by the statistical operator $\hat{\rho}_{\mathrm{ME}}$ that maximizes the von Neumann entropy $S=-\operatorname{Tr}(\hat{\rho} \ln \hat{\rho})$ subject to the constraints imposed by normalization and the expectation values $\left\langle\hat{A}_{i}\right\rangle=\operatorname{Tr}\left(\hat{\rho} \hat{A}_{i}\right)$ of the relevant observables $\hat{A}_{i}$.

Even though Jaynes' principle does provide a very satisfactory answer in many situations [17, 18], Horodecki et al [1] showed that the straightforward application of Jaynes' prescription in its usual form is not always an appropriate strategy for dealing with entangled states. It was shown in [1] that the standard implementation of Jaynes' principle may create 'fake' entanglement. For example, the MaxEnt density matrix may correspond to an entangled state even if there exist separable states compatible with the prior information. Since quantum entanglement is, in many cases, the basic resource needed when processing quantum information [1], statistical inference procedures that overestimate the amount of available entanglement should be handled with care. Furthermore, it is well known that local operations and classical communication (LOCC) can never increase the amount of entanglement between remote systems, but they can make it decrease [8]. As a consequence, one should often bet on the decrease of entanglement and not be very 'optimistic' when estimating the available amount of this resource. The above considerations suggests that, in order to deal with some situations involving entanglement, the usual form of Jaynes' prescription needs to be modified or supplemented in an appropriate way. Various such schemes have been proposed. Horodecki et al [1] proposed a combined strategy based on a constrained minimization of entanglement followed by a maximization of the von Neumann entropy. Alternatively, Abe and Rajagopal [5] explored the possibility of inferring entangled states by recourse to a variational principle based on non-extensive information measures.

Up to now, all the work done in connection with Horodecki's problem of fake inferred entanglement focused on that particular case in which the prior information is given by the mean value of the Bell operator [1,3-5]. The main purpose of the present effort is to explore what happens when the available prior information consists of the expectation value of operators exhibiting a more general form. Particular attention is going to be paid to operators nondiagonal in the Bell basis. We are going to show that the prescription proposed in [4] for solving the problem of fake entanglement is not universally applicable. We will show that there 
exist operators, both diagonal and non-diagonal in the Bell basis, for which the aforementioned prescription fails.

The paper is organized as follows. In section 2 we revisit, from a different point of view than the one employed in $[1,4,5]$, the problem of 'fake entanglement' arising when a quantum state is inferred on the basis of partial information related to the Bell observable. The inference of entangled states from prior information associated with observables non-diagonal in the Bell basis is considered in sections 3 and 4 . Finally, some conclusions are drawn in section 5 .

\section{The expectation values of the Bell observable and its square as input information}

Following Horodecki et al [1] let us assume that the prior (input) information is given by the expectation value $b$ of the Bell-CHSH observable [19]

$$
\hat{B}=\sqrt{2}\left(\sigma_{x} \otimes \sigma_{x}+\sigma_{z} \otimes \sigma_{z}\right)=2 \sqrt{2}\left(\left|\Phi^{+}\right\rangle\left\langle\Phi^{+}|-| \Psi^{-}\right\rangle\left\langle\Psi^{-}\right|\right)
$$

which is defined in terms of the components of the well known Bell basis,

$$
\begin{aligned}
& \left|\Phi^{\mp}\right\rangle=\frac{1}{\sqrt{2}}(|11\rangle \mp|00\rangle) \\
& \left|\Psi^{ \pm}\right\rangle=\frac{1}{\sqrt{2}}(|10\rangle \pm|01\rangle) .
\end{aligned}
$$

The Bell observable is nonlocal. In order to measure the Bell observable one cannot rely just upon LOCC between the parts (that is, LOCC operations). It cannot be measured without interchange of quantum information between the observers [1].

The MaxEnt state obtained by recourse to the standard prescription, when the sole available information is given by $b=\langle\hat{B}\rangle$, is described by the density matrix [1]

$$
\begin{aligned}
\hat{\rho}_{\mathrm{ME}}(b)=\frac{1}{4}[ & \left(1+\frac{b}{\sqrt{2}}+\frac{b^{2}}{8}\right)\left|\Phi^{+}\right\rangle\left\langle\Phi^{+}\left|+\left(1-\frac{b}{\sqrt{2}}+\frac{b^{2}}{8}\right)\right| \Psi^{-}\right\rangle\left\langle\Psi^{-}\right| \\
& \left.+\left(1-\frac{b^{2}}{8}\right)\left(\left|\Psi^{+}\right\rangle\left\langle\Psi^{+}|+| \Phi^{-}\right\rangle\left\langle\Phi^{-}\right|\right)\right] .
\end{aligned}
$$

Rajagopal [4] and Abe and Rajagopal [5] showed that the inclusion of $\sigma^{2}=\left\langle\hat{B}^{2}\right\rangle$ within the input data set entails important consequences for the inference of entangled states. The main idea of Rajagopal's proposal [4] is to consider the density matrix $\hat{\rho}_{\text {MS }}$ obtained by considering both mean values $b=\langle\hat{B}\rangle$ and $\sigma^{2}=\left\langle\hat{B}^{2}\right\rangle$ as constraints in the MaxEnt prescription, and assuming that the mean value of $\hat{B}^{2}$ adopts the minimum value compatible with the given value of $b$. Rajagopal proved that $\hat{\rho}_{\mathrm{MS}}$ is separable if and only if $b<\sqrt{2}$. The method employed by Rajagopal to characterize the states $\hat{\rho}_{\mathrm{MS}}$ of minimum- $\sigma^{2}$ rests heavily on the particular form of the Bell operator. A different approach is needed if one wants to implement Rajagopal's inference scheme when the input information consists of the mean value of more general observables. It is convenient now to briefly revisit the example corresponding to the Bell observable in order to (i) illustrate the viewpoint that we are going to adopt when considering more general situations, and (ii) clarify the relationships between the results we are going to report in this paper and those previously discussed in the literature.

The operators $\hat{B}$ and $\hat{B}^{2}$ verify the relations

$$
\begin{aligned}
\hat{B}^{2} & =16\left|\Phi^{+}\right\rangle\left\langle\Phi^{+}\right|-2 \sqrt{2} \hat{B} \\
& =16\left|\Psi^{-}\right\rangle\left\langle\Psi^{-}\right|+2 \sqrt{2} \hat{B} .
\end{aligned}
$$

It is easy to see, computing the trace of the above equations, that

$$
\sigma^{2} \geqslant 2 \sqrt{2}|b|
$$


and, consequently, the minimum value of $\sigma^{2}$ compatible with a given value of $b$ is

$$
\sigma^{2}=2 \sqrt{2}|b| \text {. }
$$

From the trace of equation (4) it also transpires that density matrices with the minimum value of $\sigma^{2}$ compatible with a given value of $b$ comply with

$$
\begin{array}{ll}
\left\langle\Phi^{+}|\hat{\rho}| \Phi^{+}\right\rangle=0 & \text { (if } b<0) \\
\left\langle\Psi^{-}|\hat{\rho}| \Psi^{-}\right\rangle=0 & \text { (if } b>0) .
\end{array}
$$

This means that a state complying with the minimum uncertainty requirement belongs to the three-dimensional subspace spanned by the vectors $\left\{\left|\Psi^{+}\right\rangle,\left|\Psi^{-}\right\rangle,\left|\Phi^{-}\right\rangle\right\}(b<0)$, or by the vectors $\left\{\left|\Psi^{+}\right\rangle,\left|\Phi^{+}\right\rangle,\left|\Phi^{-}\right\rangle\right\}(b>0)$. For the density matrices defined within this subspaces we have

$$
\begin{aligned}
& b=-2 \sqrt{2}\left\langle\Psi^{-}|\hat{\rho}| \Psi^{-}\right\rangle \quad \text { (if } b<0 \text { ) } \\
& b=2 \sqrt{2}\left\langle\Phi^{+}|\hat{\rho}| \Phi^{+}\right\rangle \quad \text { (if } b>0 \text { ). }
\end{aligned}
$$

The matrices provided by Rajagopal's scheme are

$$
\begin{array}{ll}
\hat{\rho}_{\mathrm{MS}}=\frac{-b}{2 \sqrt{2}}\left|\Psi^{-}\right\rangle\left\langle\Psi^{-}\right|+\frac{1}{2}\left(1+\frac{b}{2 \sqrt{2}}\right)\left[\left|\Psi^{+}\right\rangle\left\langle\Psi^{+}|+| \Phi^{-}\right\rangle\left\langle\Phi^{-}\right|\right] & (\text {if } b<0) \\
\hat{\rho}_{\mathrm{MS}}=\frac{b}{2 \sqrt{2}}\left|\Phi^{+}\right\rangle\left\langle\Phi^{+}\right|+\frac{1}{2}\left(1-\frac{b}{2 \sqrt{2}}\right)\left[\left|\Psi^{+}\right\rangle\left\langle\Psi^{+}|+| \Phi^{-}\right\rangle\left\langle\Phi^{-}\right|\right] \quad(\text { if } b>0) .
\end{array}
$$

States that are diagonal in the Bell basis (2) are separable if and only if they have no eigenvalue larger than $1 / 2$ [1]. Hence, it follows from equation (9) that the states $\hat{\rho}_{\mathrm{MS}}$ are separable if and only if $|b|<\sqrt{2}$.

Let us now consider general minimum uncertainty states (that is, states $\hat{\rho}$ verifying (6) but not necessarily of the MaxEnt form). Expressing the matrix elements of $\hat{\rho}$ in the Bell basis (2), let us equate all the non-diagonal elements to zero and leave unchanged the diagonal ones. The new density matrix $\hat{\rho}_{\mathrm{D}}$ thus obtained has always less entanglement than the original $\hat{\rho}$ [1]. If the original $\hat{\rho}$ is such that $b>\sqrt{2}$, then the matrix $\hat{\rho}_{\mathrm{D}}$ (which is diagonal in the Bell basis) will have one eigenvalue greater than $1 / 2$ (see equation (8)). Thus, $\hat{\rho}_{\mathrm{D}}$ is entangled and so is $\hat{\rho}$. Summing up, there is no separable density matrix complying with the minimum$\sigma^{2}$ condition (6) and having $b>\sqrt{2}$. This means that, for $b>\sqrt{2}$, the inference scheme proposed by Rajagopal does not produce 'fake' inferred entanglement. At least when the input data is related to the Bell observable (1), Rajagopal's prescription does not lead to an entangled inferred state $\hat{\rho}_{\mathrm{MS}}$ if there are separable states compatible with the constraints $b$ and $\sigma^{2}$. This is the main result obtained by Rajagopal $[4,5]$, although he arrived to it by recourse to a different line of reasoning.

Quantitative measures of entanglement constitute interesting tools for studying the entanglement-related properties exhibited by the standard MaxEnt scheme and other statistical inference methods. Notice that both Horodecki's and Rajagopal's discussions of the problem of fake inferred entanglement only distinguish between separable and entangled states. No degree of entanglement is thereby ascertained. However, as is well known, entangled states differ in the amount of entanglement they have. A quantitative measure of entanglement enables us to compare the degree of entanglement of both (i) the inferred quantum state $\hat{\rho}_{\text {inferred }}$ yielded by an inference scheme when only partial information is available about the 'true' state $\hat{\rho}_{\text {true }}$ of the system and (ii) the entanglement of $\hat{\rho}_{\text {true }}$. When both states $\hat{\rho}_{\text {inferred }}$ and $\hat{\rho}_{\text {true }}$ are entangled, we would like to know the amount of entanglement that each of these statistical operators carries with it. A physically motivated measure of entanglement is provided by the entanglement of formation $E[\hat{\rho}]$ [20]. This measure quantifies the resources needed to create a given entangled 


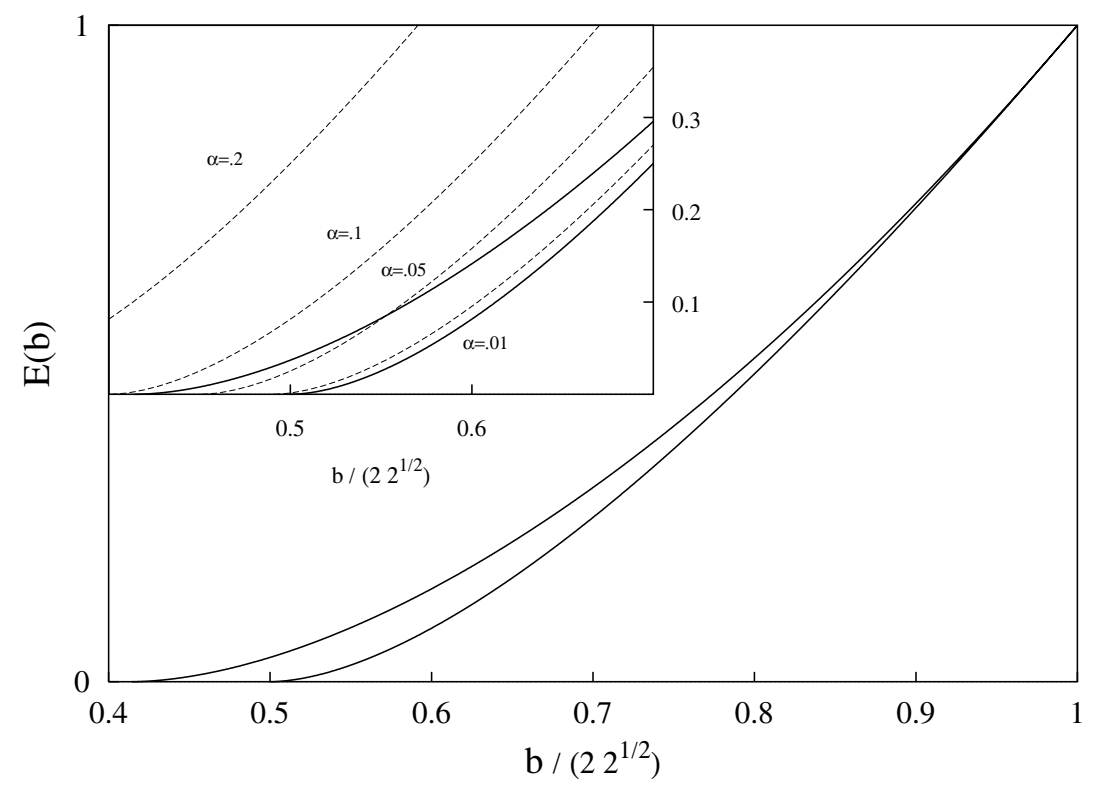

Figure 1. The entanglement of formation $E[\hat{\rho}]$, as a function of (i) the expectation value $b$ of the Bell operator, (ii) the MaxEnt density matrix $\hat{\rho}_{\mathrm{ME}}$ (equation (3)) (upper solid curve), and (iii) the minimum- $\sigma^{2}$ density matrix $\hat{\rho}_{\mathrm{MS}}$ (equation (9)) (lower solid curve). The results corresponding to the density matrix ansatz (14) (dashed curves) are shown in the inset.

state $\hat{\rho}$. As explained in $[8,20], E[\hat{\rho}]$ is equal to the asymptotic limit (for large $n$ ) of a certain quotient $m / n$. Here $m$ is the number of singlet states needed to create $n$ copies of the state $\hat{\rho}$ when the optimum procedure based on local operations is employed. Obviously, the entanglement of formation of a separable state is equal to zero, that is $E\left(\hat{\rho}_{\text {sep. }}\right)=0$. For the particular case of two-qubits states, Wootters obtained an explicit expression for $E[\hat{\rho}]$ in terms of the density matrix $\hat{\rho}$ [21]. Wootters' formula reads [21]

$$
E[\hat{\rho}]=h\left(\frac{1+\sqrt{1-C^{2}}}{2}\right)
$$

where

$$
h(x)=-x \log _{2} x-(1-x) \log _{2}(1-x)
$$

and $C$ stands for the so-called concurrence of the two-qubits state $\hat{\rho}$. The concurrence is given by

$$
C=\max \left(0, \lambda_{1}-\lambda_{2}-\lambda_{3}-\lambda_{4}\right)
$$

$\lambda_{i}, \quad(i=1, \ldots, 4)$ being the square roots, in decreasing order, of the eigenvalues of the matrix $\hat{\rho} \tilde{\rho}$, with

$$
\tilde{\rho}=\left(\sigma_{y} \otimes \sigma_{y}\right) \rho^{*}\left(\sigma_{y} \otimes \sigma_{y}\right)
$$

The above expression is to be evaluated by recourse to the matrix elements of $\hat{\rho}$ computed with respect to the product basis.

Figure 1 depicts the entanglement of formation as a function of the input data $b$ (for $b>0$ ). Two types of inferred density matrix are used to compute the entanglement of formation, namely (i) the density matrix $\hat{\rho}_{\mathrm{ME}}$ yielded by the standard MaxEnt procedure (upper solid 
curve) and (ii) the density matrix $\hat{\rho}_{\text {MS }}$ provided by Rajagopal's minimum- $\sigma^{2}$ scheme (lower solid curve). form

Let us suppose that the 'true' state of the system is described by a density matrix of the

$$
\begin{aligned}
& \hat{\rho}_{\mathrm{T}}(\alpha)=\left(\frac{b}{2 \sqrt{2}}+\alpha\right)\left|\Phi^{+}\right\rangle\left\langle\Phi^{+}|+\alpha| \Psi^{-}\right\rangle\left\langle\Psi^{-}\right| \\
& +\frac{1}{2}\left(1-\frac{b}{2 \sqrt{2}}-2 \alpha\right)\left(\left|\Phi^{-}\right\rangle\left\langle\Phi^{-}|+| \Psi^{+}\right\rangle\left\langle\Psi^{+}\right|\right) .
\end{aligned}
$$

The ('true') density matrices belonging to the above family are characterized by a parameter $\alpha$ and verify $\operatorname{Tr}\left(\hat{\rho}_{\mathrm{T}} \hat{\boldsymbol{B}}\right)=b$. We assume that the only knowledge we have about $\hat{\rho}_{\mathrm{T}}$ is given by the mean value $b$. From this piece of data we can determine the inferred matrices $\hat{\rho}_{\text {ME }}$ and $\hat{\rho}_{\text {MS }}$ provided, respectively, by the standard MaxEnt and Rajagopal's prescriptions. In the inset of figure 1 we can see, together with the entanglement of formation of both $\hat{\rho}_{\mathrm{ME}}$ and $\hat{\rho}_{\mathrm{MS}}$, the behaviour (as a function of $b$ ) of the entanglement of formation $E\left[\hat{\rho}_{\mathrm{T}}(\alpha)\right]$, i.e. that of the 'true' state.

We believe that the $(b, E(b))$-plane depicted in figure 1, representing input information $b$ versus the inferred entanglement $E(b)$, constitutes a useful device for visualizing the entanglement-related properties of an inference scheme. In figure 1 we can compare how both the standard MaxEnt scheme, and the one advanced by Rajagopal, behave in the $(b, E(b))$ plane. The most noteworthy feature of figure 1 is that (when the input information is related to the Bell observable) the results obtained using the usual MaxEnt method do not seem to differ too much from those obtained using Rajagopal's prescription.

\section{Input information associated with observables non-diagonal in the Bell basis}

As already mentioned, both Horodecki and Rajagopal treatments of the problem of fake inferred entanglement focused on the case of prior knowledge related to the Bell observable. We want to explore here to what extent the conclusions reached by those researchers are valid when the available prior information consists on the expectation values of more general observables. In particular, we want to explore what happens when observables non-diagonal in the Bell basis are considered. As we shall presently see, an interesting example illustrating new aspects of the phenomenon of fake entanglement is provided by the quantum observable associated with the Hermitian operator

$$
\hat{A}=\kappa(|1\rangle\langle 1|+| 3\rangle\langle 3|)+\lambda|2\rangle\langle 2|
$$

where $\kappa$ and $\lambda$ are real parameters such that

$$
\kappa \geqslant 0 \geqslant \lambda
$$

and whose eigenvectors $|i\rangle(i=1, \ldots, 4)$ are

$$
\begin{aligned}
|1\rangle & =\frac{1}{\sqrt{2}}(|11\rangle+|00\rangle) \\
|2\rangle & =\frac{1}{\sqrt{2}}(|11\rangle-|00\rangle) \\
|3\rangle & =|01\rangle \\
|4\rangle & =|10\rangle .
\end{aligned}
$$

It is clear that $\hat{A}$ is non-diagonal in the Bell basis. The observable $\hat{A}$ is nonlocal. It cannot be measured without interchange of quantum information between the observers. Consequently, 
and as far as its nonlocality properties are concerned, the observable $\hat{A}$ has the same status as the Bell observable considered by Horodecki [1], Rajagopal [4] and Abe and Rajagopal [5]. Sancho and Huelga [6] recently proved that the knowledge of the expectation value of just one observable (even if the observable is nonlocal) is not enough to determine completely the amount of entanglement of a given, unknown, bipartite pure state. This important result immediately raises the question of how reliably the entanglement of an unknown quantum state can be inferred from the sole knowledge of the mean value of a nonlocal observable. We are going to explore here some aspects of this question, mainly in connection with the problem of fake inferred entanglement. Let us suppose that we know the expectation value $a$ of $\hat{A}$, given by

$$
a=\operatorname{Tr}(\hat{\rho} \hat{A})=\kappa(\langle 1|\hat{\rho}| 1\rangle+\langle 3|\hat{\rho}| 3\rangle)+\lambda\langle 2|\hat{\rho}| 2\rangle .
$$

Following the proposal first advanced in $[4]$ (see also $[3,5])$ we are going to incorporate a new constraint associated with the expectation value of

$$
\hat{A}^{2}=\kappa^{2}(|1\rangle\langle 1|+| 3\rangle\langle 3|)+\lambda^{2}|2\rangle\langle 2|
$$

which is

$$
\sigma^{2}=\operatorname{Tr}\left(\hat{\rho} \hat{A}^{2}\right)=\kappa^{2}(\langle 1|\hat{\rho}| 1\rangle+\langle 3|\hat{\rho}| 3\rangle)+\lambda^{2}\langle 2|\hat{\rho}| 2\rangle .
$$

According to the strategy suggested in [4], the problem of fake inferred entanglement can be solved if in order to describe our system we adopt a density matrix $\hat{\rho}_{\text {MS }}$ complying with two requisites. First, $\hat{\rho}_{\text {MS }}$ must have the MaxEnt form corresponding to the constraints associated with the expectation values of both $\hat{A}$ and $\hat{A}^{2}$. Secondly, the expectation value $\sigma^{2}$ must adopt the lowest value compatible with the given value of $a$. Notice that the mean value $a=\langle\hat{A}\rangle$ is the only independent input data. For the sake of simplicity we are going to restrict our considerations to the case of positive values of $\langle\hat{A}\rangle$.

The mean values of $\hat{A}$ and $\hat{A}^{2}$ are related by

$$
\sigma^{2}=\kappa a+\lambda(\lambda-\kappa)\langle 2|\hat{\rho}| 2\rangle
$$

which implies that those mixed states characterized by exhibiting the minimum possible $\sigma^{2}$ value compatible with a given $a>0$ must verify $\langle 2|\hat{\rho}| 2\rangle=0$. Consequently, for those states with minimum- $\sigma^{2}$ we have

$$
\sigma^{2}=\kappa a \text {. }
$$

When we have a single constraint corresponding to the mean value of $\hat{A}$, the MaxEnt density matrix is

$$
\hat{\rho}_{\mathrm{ME}}^{I}=\frac{1}{Z} \exp (-\beta \hat{A})
$$

where $\beta$ is a Lagrange multiplier and $Z=\operatorname{Tr}(\exp (-\beta \hat{A}))$. Alternatively, $\hat{\rho}_{\mathrm{ME}}^{I}$ can be cast as

$$
\hat{\rho}_{\mathrm{ME}}^{I}=\frac{1}{1+2 w+w^{\lambda / \kappa}}\left[w(|1\rangle\langle 1|+| 3\rangle\langle 3|)+w^{\lambda / \kappa}|2\rangle\langle 2|+| 4\rangle\langle 4|\right]
$$

where $w=\exp (-\beta \kappa)$ verifies

$$
\frac{a}{\kappa}=\frac{2 w+(\lambda / \kappa) w^{\lambda / \kappa}}{1+2 w+w^{\lambda / \kappa}} .
$$

The MaxEnt statistical operator associated with the expectation values $a$ and $\sigma^{2}$ as input information is

$$
\hat{\rho}_{\mathrm{ME}}^{I I}=\frac{1}{Z} \exp \left(-\beta \hat{A}-\gamma \hat{A}^{2}\right)
$$


where $\beta$ and $\gamma$ are appropriate Lagrange multipliers and the partition function $Z$ is given by

$$
Z=\operatorname{Tr}\left(\exp \left(-\beta \hat{A}-\gamma \hat{A}^{2}\right)\right) .
$$

The matrix $\hat{\rho}_{\mathrm{ME}}^{I I}$ can be expressed explicitly in terms of the input mean values $a$ and $\sigma^{2}$,

$\hat{\rho}_{\mathrm{ME}}^{I I}=\frac{1}{2} \frac{\sigma^{2}-\lambda a}{\kappa(\kappa-\lambda)}(|1\rangle\langle 1|+| 3\rangle\langle 3|)+\frac{\kappa a-\sigma^{2}}{\lambda(\kappa-\lambda)}|2\rangle\left\langle 2\left|+\frac{\sigma^{2}-a(\kappa+\lambda)+\lambda \kappa}{\lambda \kappa}\right| 4\right\rangle\langle 4|$.

When the further requirement of a minimum value for $\sigma^{2}$ is imposed, the above MaxEnt density matrix reduces to

$$
\hat{\rho}_{\mathrm{MS}}=\frac{a}{2 \kappa}(|1\rangle\langle 1|+| 3\rangle\langle 3|)+\left(1-\frac{a}{\kappa}\right)|4\rangle\langle 4| .
$$

Since we always have $\kappa \geqslant a$, the above matrix is positive semidefinite.

Now, in order to find out whether Rajagopal's prescription is plagued with the problem of fake inferred entanglement (when applied in connection with the observable $\hat{A}$ ), we need to proceed according to what follows. First, we adopt a form for the 'true' density matrix describing the system. Second, we assume that the only available information about the true state consists on the expectation value of $\hat{A}$. From this sole piece of data we obtain, via the inference scheme we are studying, the inferred density matrix. Finally, we compare the entanglement properties associated with the original, true density matrix with the entanglement properties exhibited by the inferred one. In particular, we can evaluate on both matrices an appropriate quantitative measure of entanglement. In what follows we are going to assume that the true state of the system is described by an statistical operator belonging to the family of density matrices

$$
\hat{\rho}_{\mathrm{S}}=p|1\rangle\langle 1|+\alpha| 3\rangle\langle 3|+(1-p-\alpha)| 4\rangle\langle 4|
$$

where $p$ and $\alpha$ are real positive parameters verifying

$$
\begin{aligned}
& 0 \leqslant p \leqslant 1 \\
& 0 \leqslant \alpha \leqslant 1-p .
\end{aligned}
$$

Notice that the 'true' density matrices (30) that we are trying to infer by recourse to different schemes are not of the MaxEnt form, nor of the form associated with any other statistical inference scheme. The expectation values of $\hat{A}$ and $\hat{A}^{2}$, evaluated on $\hat{\rho}_{\mathrm{S}}$ are

$$
a=p \kappa+\alpha \kappa
$$

and

$$
\sigma^{2}=p \kappa^{2}+\alpha \kappa^{2}
$$

Suppose we are given the expectation values $a$ and $\sigma^{2}$ corresponding to a given state belonging to the family (30) (notice that, for this family of density matrices, the mean values $a$ and $\sigma^{2}$ always verify the minimum- $\sigma^{2}$ condition (22)). We can take those mean values as input information and generate the concomitant inferred density matrix. That is, we can associate a MaxEnt state to each member of (30). The performance of the inference scheme can be studied by comparing the entanglement properties of a member of the parametrized family (30) with those of the concomitant inferred state. As a first step we are going to find out, by recourse to Peres' separability criterion [22], whether there are separable states of the form (30) leading to entangled inferred states. Peres' criterion is based on a partial transposition transformation [22]. To be more specific, let the density matrix elements (with respect to a product basis) of a statistical operator $\hat{\rho}$ be

$$
\rho_{m \mu, n v}=\langle m \mu|\hat{\rho}| n v\rangle
$$


where Latin indices refer to the first subsystem and Greek indices to the second one. The partial transpose $\hat{\rho}^{\mathrm{PT}}$ of $\hat{\rho}$ is a matrix whose elements are obtained by the partial transposition of the elements of $\hat{\rho}$, i.e.

$$
\hat{\rho}_{m \mu, n \nu}^{\mathrm{PT}}=\hat{\rho}_{n \mu, m \nu} .
$$

It can be shown that $\hat{\rho}$ is separable if and only if $\hat{\rho}^{\mathrm{PT}}$ has no negative eigenvalues [23]. If we apply the Peres criterion to the minimum- $\sigma^{2}$ MaxEnt density matrix $\hat{\rho}_{\text {MS }}$ (equation (29)) we find that there is only one eigenvalue of the partial transpose matrix that may adopt negative values. This eigenvalue is

$$
\delta=-\frac{a}{4 \kappa}+\frac{1}{2}-\frac{1}{4} \sqrt{\frac{a}{\kappa}\left(10 \frac{a}{\kappa}-12\right)+4} .
$$

Hence, we have

$$
\begin{aligned}
& a / \kappa \leqslant 8 / 9 \Longleftrightarrow \delta \geqslant 0 \\
& a / \kappa>8 / 9 \Longleftrightarrow \delta<0 .
\end{aligned}
$$

Consequently, $\hat{\rho}_{\mathrm{MS}}$ is separable if $a / \kappa \leqslant 8 / 9$ and entangled otherwise. Using the Peres criterion we can also determine just when the parametrized (true) density matrix $\hat{\rho}_{\mathrm{S}}$ is separable. For the considerations that follow it will prove convenient to rewrite $\hat{\rho}_{\mathrm{S}}$ in terms of the expectation value $a=\operatorname{Tr}\left(\hat{\rho}_{\mathrm{S}} \hat{A}\right)$,

$$
\hat{\rho}_{\mathrm{S}}=\left(\frac{a}{\kappa}-\alpha\right)|1\rangle\langle 1|+\alpha| 3\rangle\left\langle 3\left|+\left(1-\frac{a}{\kappa}\right)\right| 4\right\rangle\langle 4| .
$$

It is important to stress that the above expression describes the same family of mixed states defined by equation (30). The states $\hat{\rho}_{\mathrm{S}}$ associated with equation (38) still depend on two independent parameters, i.e. $\alpha$ and $a / \kappa$. Equation (38) is just a re-parametrization of the family (30) where, for the sake of convenience, we have chosen $a / \kappa=\operatorname{Tr}\left(\hat{\rho}_{\mathrm{S}} \hat{A}\right) / \kappa$ as one of the two relevant parameters. The separability of $\hat{\rho}_{\mathrm{S}}$ is determined by the quantity

$$
Q=\frac{1}{2}-\frac{a}{2 \kappa}+\frac{\alpha}{2}-\frac{1}{2} \sqrt{2\left(\frac{a}{\kappa}\right)^{2}-\frac{2 a}{\kappa}+1-2 \alpha+2 \alpha^{2}}
$$

The statistical operator $\hat{\rho}_{\mathrm{S}}$ is separable if $Q \geqslant 0$ and entangled otherwise. The boundaries (in the plane $(\alpha, a)$ ) between the separability and the entangled regions corresponding to (i) the density operators $\hat{\rho}_{\mathrm{S}}$, (ii) the standard MaxEnt statistical operators $\hat{\rho}_{\mathrm{ME}}^{I}$ and (iii) the minimum$\sigma^{2}$ MaxEnt density matrices $\hat{\rho}_{\mathrm{MS}}$ are depicted in figure 2 , where we take $\kappa=1$ and $\lambda=-1$. Notice that only those points with $\alpha<a$ are physically meaningful, since $(\alpha, a)$ pairs not subject to that inequality lead to a matrix $\hat{\rho}_{S}$ with one negative eigenvalue. Figure 2 is to be interpreted as follows. There are three density matrices associated with each point in the plane $(\alpha, a)$ :

- The ('true') $\hat{\rho}_{\mathrm{S}}$ matrix given by the expression (38).

- The (inferred) density matrix $\hat{\rho}_{\mathrm{ME}}^{I}$, of the standard MaxEnt form (23), (24).

- The (inferred) density matrix $\hat{\rho}_{\mathrm{MS}}$ of the minimum- $\sigma^{2}$ MaxEnt form (29).

For all the three aforementioned density matrices the expectation value of $\hat{A}$ is $a$ (that is, $\left.a=\operatorname{Tr}\left(\hat{\rho}_{\mathrm{MS}} \hat{A}\right)=\operatorname{Tr}\left(\hat{\rho}_{\mathrm{ME}}^{I}\right)=\operatorname{Tr}\left(\hat{\rho}_{\mathrm{S}} \hat{A}\right)\right)$. The density matrix $\hat{\rho}_{\mathrm{MS}}$ is the one yielded by Rajagopal's prescription if one tries to infer $\hat{\rho}_{\mathrm{S}}$ from the sole knowledge of the expectation value $a=\operatorname{Tr}\left(\hat{\rho}_{\mathrm{S}} \hat{A}\right)$. The standard MaxEnt procedure, instead, would lead to $\hat{\rho}_{\mathrm{ME}}^{I}$. Using the Peres criterion we can determine when the inferred density matrix $\hat{\rho}_{\mathrm{ME}}^{I}$ is entangled. For $\kappa=1$ and $\lambda=-1$ we found that $\hat{\rho}_{\mathrm{ME}}^{I}$ is separable when $a \leqslant 0.8564$ and entangled otherwise. The lines $l$ and $m$ in figure 2 correspond to $a=0.8564$ and $a=8 / 9$, respectively. The 


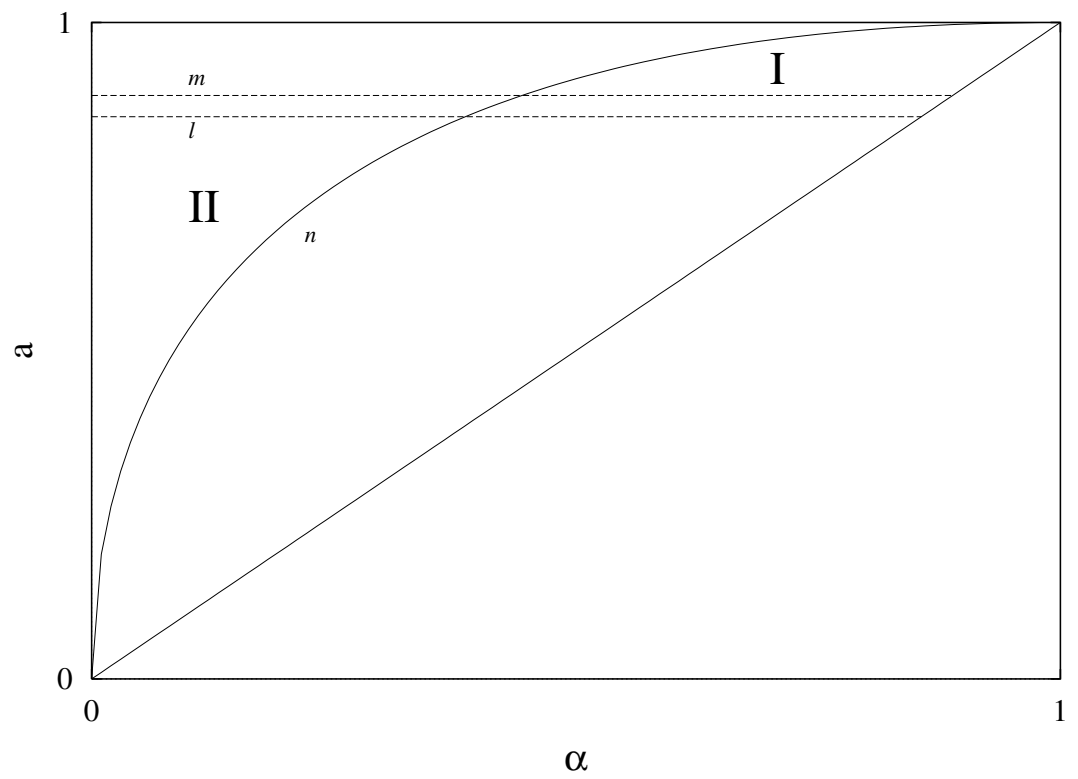

Figure 2. Boundaries between the regions corresponding to separability and entanglement for states described by the density matrices $\hat{\rho}_{\mathrm{ME}}^{I}($ line $l), \hat{\rho}_{\mathrm{MS}}\left(\right.$ line $m$ ) and $\hat{\rho}_{\mathrm{S}}($ line $n)$. The expressions for the matrices $\hat{\rho}_{\mathrm{ME}}^{I}, \hat{\rho}_{\mathrm{MS}}$ and $\hat{\rho}_{\mathrm{S}}$ are given, respectively, by equations (24), (29) and (38).

curve $n$ represents the equation $Q(a, \alpha)=0$. The density matrices $\hat{\rho}_{\mathrm{ME}}\left(\hat{\rho}_{\mathrm{MS}}\right)$ are entangled for points $(a, \alpha)$ lying above the line $l(m)$ and separable otherwise. On the other hand, the matrices $\hat{\rho}_{\mathrm{S}}$ are separable when $(a, \alpha)$ lies below the curve $n$ and entangled if $(a, \alpha)$ lies above $n$. Of particular interest are the regions I and II. In region I the ('true') density matrix to be inferred, $\hat{\rho}_{\mathrm{S}}$, is separable, while the associated ('inferred') matrix $\hat{\rho}_{\mathrm{MS}}$, provided by Rajagopal's inference scheme, is not. In region II things are quite different: the inference scheme provides a separable statistical operator $\hat{\rho}_{\mathrm{MS}}$ while the matrix to be inferred, $\hat{\rho}_{\mathrm{S}}$, is entangled. It is clear that the MaxEnt minimum- $\sigma^{2}$ inference procedure advanced by Rajagopal $[4,5]$ generates fake entanglement when applied to states $\hat{\rho}_{\mathrm{S}}$ associated with points $(a, \alpha)$ belonging to region I. Contrary to previous evidence obtained when the Bell observable mean value is taken as the prior information [3-5], we must conclude that the MaxEnt minimum- $\sigma^{2}$ scheme does not provide a general solution to the problem of fake entanglement.

The comparison of the amount of entanglement of formation exhibited by the states $\hat{\rho}_{\mathrm{S}}$ and $\hat{\rho}_{\text {MS }}$ enables us to study the problem of fake inferred entanglement in a quantitative way. The curves depicted in figure 3 display the behaviour of $E\left[\hat{\rho}_{\mathrm{MS}}\right]$ and $E\left[\hat{\rho}_{\mathrm{S}}\right]$ as a function of the mean value $a$ of the observable $\hat{A}$ (again, with $\kappa=1$ and $\lambda=-1$ ). The upper solid curve corresponds to $E\left[\hat{\rho}_{\mathrm{ME}}^{I}\right]$, the lower solid curve to $E\left[\hat{\rho}_{\mathrm{MS}}\right]$, and the dashed and dot-dashed curves to $E\left[\hat{\rho}_{\mathrm{S}}\right]$, for different values of the parameter $\alpha$. The results exhibited in figure 3 illustrate how, for each given value of the input data $a=\operatorname{Tr}(\hat{\rho} \hat{A})$, the entanglement of formation $E$ of the density operators yielded by both the standard MaxEnt method $\left(\hat{\rho}_{\mathrm{ME}}^{I}\right)$ and Rajagopal's scheme $\left(\hat{\rho}_{\mathrm{MS}}\right)$ compare with the entanglement of formation of the state to be inferred $\left(\hat{\rho}_{\mathrm{S}}\right)$. It is clear from figure 3 that, with regard to the behaviour of the inferred amount of entanglement as a function of the input information (at least when this input data consists of $\langle\hat{A}\rangle$ ), the prescription advanced by Rajagopal does not appreciably differ from the standard MaxEnt result. In particular, both prescriptions tend to yield the same results in the limit $a \rightarrow 1$. 


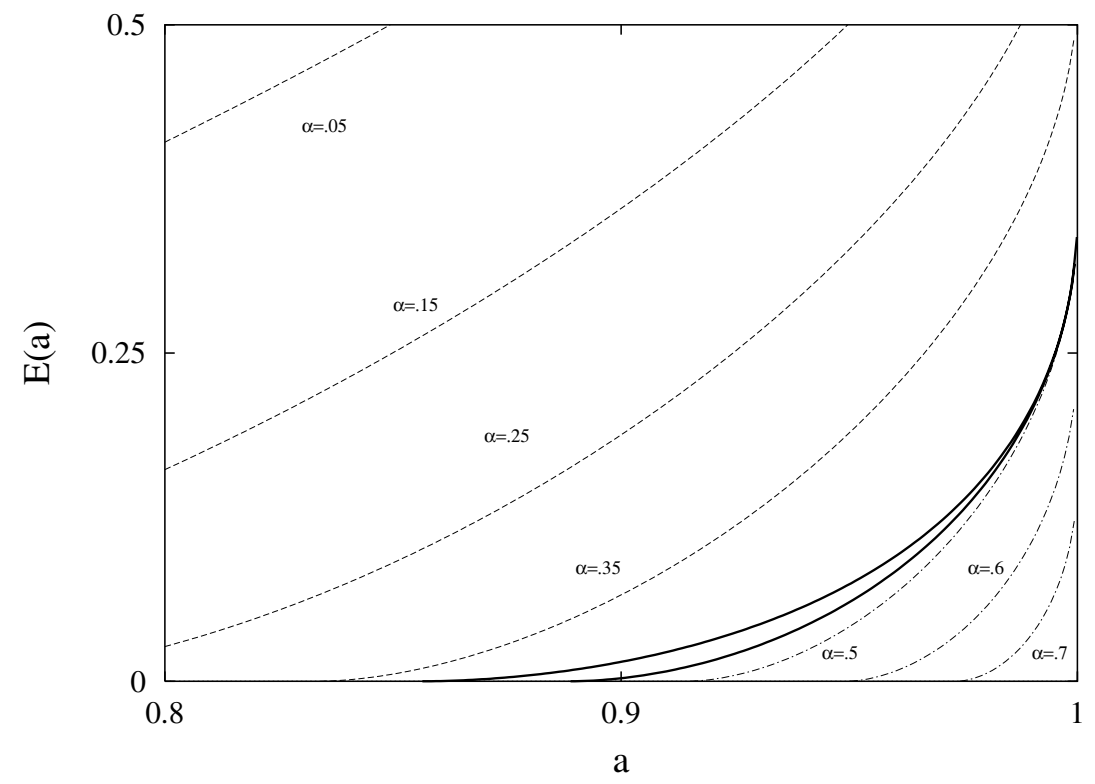

Figure 3. The entanglement of formation $E[\hat{\rho}]$ as a function the expectation value of the observable $\hat{A}$ (equation (15)) with $\kappa=1$ and $\lambda=-1$, corresponding to $\hat{\rho}_{\mathrm{ME}}^{I}$ (upper solid curve), to $\hat{\rho}_{\mathrm{MS}}$ (lower solid curve), and to $\hat{\rho}_{\mathrm{S}}$, for the values of $\alpha$ indicated in the figure (dashed and dot-dashed curves). The expressions for the matrices $\hat{\rho}_{\mathrm{ME}}^{I}, \hat{\rho}_{\mathrm{MS}}$ and $\hat{\rho}_{\mathrm{S}}$ are given, respectively, by equations (24), (29) and (38).

Notice that the MaxEnt minimum- $\sigma^{2}$ matrix $\hat{\rho}_{\mathrm{MS}}$ does not depend upon the value of $-(\lambda / \kappa)$, unlike what happens with the standard MaxEnt matrix $\hat{\rho}_{\mathrm{ME}}^{I}$. This dependence upon $-(\lambda / \kappa)$ is depicted in figure 4 , where we can appreciate the behaviour of the entanglement of formation $E[\hat{\rho}]$ as a function of $(a / \kappa)$ corresponding to (i) the density operators $\hat{\rho}_{\mathrm{MS}}$ (lower solid curve) and (ii) the MaxEnt density matrices $\hat{\rho}_{\mathrm{ME}}^{I}$ associated with different values of the ratio $-(\lambda / \kappa)$ (dashed curves). The upper solid curve in figure 4 corresponds to the particular case $-(\lambda / \kappa)=1$. The MaxEnt density matrices $\hat{\rho}_{\mathrm{ME}}^{I}$ are entangled for values of $a$ greater than a critical value $a_{\mathrm{c}}$ depending on $-(\lambda / \kappa)$. The behaviour of $\left(a_{\mathrm{c}} / \kappa\right)$ as a function of $-(\lambda / \kappa)$ is depicted in the inset of figure 4 .

\section{Prior information associated with more general observables}

In this section we are going to assume that the prior information is given by the expectation value of an observable of the form

$$
\hat{D}=|1\rangle\left\langle 1\left|+\alpha_{1}\right| 2\right\rangle\left\langle 2\left|+\alpha_{2}\right| 3\right\rangle\langle 3|
$$

with eigenvectors

$$
\begin{aligned}
& |1\rangle=\left|\Phi^{+}\right\rangle \\
& |2\rangle=\left|\Phi^{-}\right\rangle \\
& |3\rangle=\sin \theta|10\rangle+\cos \theta|01\rangle \\
& |4\rangle=\cos \theta|10\rangle-\sin \theta|01\rangle
\end{aligned}
$$

and eigenvalues $0,1, \alpha_{1}, \alpha_{2}$, such that

$$
\alpha_{2}>\alpha_{1}>1 \text {. }
$$




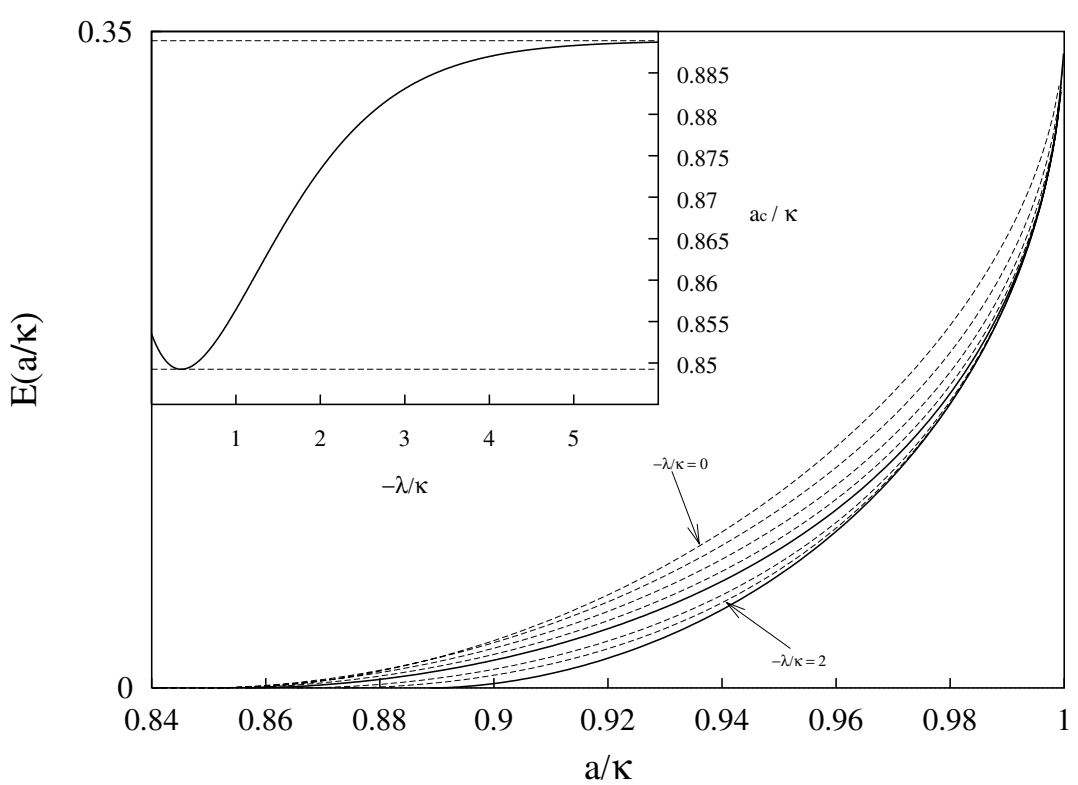

Figure 4. The entanglement of formation $E[\hat{\rho}]$ as a function of $(a / \kappa)$, where $a$ is the expectation value of the observable $\hat{A}$ (equation (15)), corresponding to $\hat{\rho}_{\mathrm{MS}}$ (lower solid curve) and to the MaxEnt density matrices $\hat{\rho}_{\mathrm{ME}}^{I}$ associated with different values of the ratio $-(\lambda / \kappa)$ (dashed curves). The upper solid curve corresponds to the particular case $-(\lambda / \kappa)=1$. The expressions for the matrices $\hat{\rho}_{\mathrm{ME}}^{I}$ and $\hat{\rho}_{\mathrm{MS}}$ are given, respectively, by equations (24) and (29). The critical values $\left(a_{\mathrm{c}} / \kappa\right)$ where the matrices $\hat{\rho}_{\mathrm{ME}}^{I}$ begin to be entangled are depicted in the inset as a function of $-(\lambda / \kappa)$.

The operator $\hat{D}$ cannot be measured using only LOCC operations. In this respect it behaves like both the Bell observable and the observable $\hat{A}$ introduced in the preceding section.

The mean values $d=\langle\hat{D}\rangle$ and $\sigma^{2}=\left\langle\hat{D}^{2}\right\rangle$ are related by

$$
\sigma^{2}-d=\alpha_{1}\left(\alpha_{1}-1\right)\langle 2|\hat{\rho}| 2\rangle+\alpha_{2}\left(\alpha_{2}-1\right)\langle 3|\hat{\rho}| 3\rangle .
$$

In order to apply the inference method advanced by Rajagopal we need first to determine the form adopted by the statistical operators $\hat{\rho}$ characterized by the minimum possible value of $\sigma^{2}$ compatible with a given value of $d$. As we will presently see, the particular form exhibited by the minimum- $\sigma^{2}$ density matrices depends on the value of the constraint $d$. It is clear from (40) and (42) that $0 \leqslant d \leqslant \alpha_{2}$. The minimum- $\sigma^{2}$ matrices adopt three different forms associated, respectively, with $d$-values belonging to the intervals [0,1], [1, $\left.\alpha_{1}\right]$ and $\left[\alpha_{1}, \alpha_{2}\right]$. It follows from (43) that

$$
0 \leqslant d \leqslant 1 \Rightarrow \hat{\rho}_{\mathrm{MS}}=d|1\rangle\langle 1|+(1-d)| 4\rangle\langle 4| .
$$

In order to analyse the case corresponding to $d \in\left[1, \alpha_{1}\right]$ it will prove convenient to introduce the definitions

$$
\begin{aligned}
& p=\langle 1|\hat{\rho}| 1\rangle \\
& S=\langle 2|\hat{\rho}| 2\rangle+\langle 3|\hat{\rho}| 3\rangle \\
& s_{1}=\langle 2|\hat{\rho}| 2\rangle / S \\
& s_{2}=\langle 3|\hat{\rho}| 3\rangle / S .
\end{aligned}
$$


All the above quantities belong to the interval [0,1]. Furthermore, we have $s_{1}+s_{2}=1$ and $0 \leqslant p+S \leqslant 1$. The expectation value of $\hat{D}$ is given by

$$
d=\operatorname{Tr}(\hat{\rho} \hat{D})=p+S\left(s_{1} \alpha_{1}+s_{2} \alpha_{2}\right)
$$

and the minimization of $\sigma^{2}$ is equivalent to finding the minimum value of the quantity

$$
M=S\left(s_{1} \alpha_{1}\left(\alpha_{1}-1\right)+s_{2} \alpha_{2}\left(\alpha_{2}-1\right)\right) .
$$

The variables $p, S$, and $s_{1}$ verify

$$
0 \leqslant p+S=d-S\left(s_{1}\left(\alpha_{1}-1\right)+\left(1-s_{1}\right)\left(\alpha_{2}-1\right)\right) \leqslant 1 .
$$

Notice that once a particular value of $d$ is fixed the parameters $p, S$, and $s_{1}$ are no longer independent quantities: they are related by (46) (which is equivalent to the equality relation in (48)). Regarding $S$ and $s_{1}$ as independent quantities, the optimization problem we have to solve is to find the pair of numbers $\left(S, s_{1}\right)$ belonging to $[0,1]$ that, complying with the inequalities in (48), make $M$ a minimum. If we are given a pair $\left(S, s_{1}\right)$ satisfying the aforementioned requisites, it is clear that we can decrease $S$ until the last inequality in (48) becomes an equality. Hence, the optimum $\left(S, s_{1}\right)$ must verify

$$
S=\frac{d-1}{s_{1}\left(\alpha_{1}-1\right)+\left(1-s_{1}\right)\left(\alpha_{2}-1\right)}
$$

and $M$ can be rewritten as a function of the sole variable $s_{1}$ (recall that $s_{2}=1-s_{1}$ )

$$
M=(d-1) \frac{s_{1} \alpha_{1}\left(\alpha_{1}-1\right)+s_{2} \alpha_{2}\left(\alpha_{2}-1\right)}{s_{1}\left(\alpha_{1}-1\right)+\left(1-s_{1}\right)\left(\alpha_{2}-1\right)} .
$$

Notice that (49) determines a value of $S$ that, for any value of $s_{1} \in[0,1]$, belongs to the interval $[0,1]$. Introducing now the quantities

$$
\begin{aligned}
& t_{1}=s_{1}\left(\alpha_{1}-1\right) /\left[s_{1}\left(\alpha_{1}-1\right)+\left(1-s_{1}\right)\left(\alpha_{2}-1\right)\right] \\
& t_{2}=\left(1-s_{1}\right)\left(\alpha_{2}-1\right) /\left[s_{1}\left(\alpha_{1}-1\right)+\left(1-s_{1}\right)\left(\alpha_{2}-1\right)\right]
\end{aligned}
$$

the function $M$ to be minimized can be cast under the guise

$$
M=(d-1)\left(t_{1} \alpha_{1}+t_{2} \alpha_{2}\right)
$$

which clearly adopts its minimum value when $t_{1}=1$ and $t_{2}=0$. That is, the minimum obtains when $s_{1}=1$. Summing up, the minimum- $\sigma^{2}$ density matrix compatible with a given value of $d \in\left[1, \alpha_{1}\right]$ corresponds to

$$
\begin{aligned}
& S=\frac{d-1}{\alpha_{1}-1} \\
& s_{1}=1 .
\end{aligned}
$$

The concomitant density operator reads

$$
\hat{\rho}_{\mathrm{MS}}=\left(\frac{\alpha_{1}-d}{\alpha_{1}-1}\right)|1\rangle\left\langle 1\left|+\left(\frac{d-1}{\alpha_{1}-1}\right)\right| 2\right\rangle\langle 2| .
$$

A similar reasoning can be applied in order to obtain $\hat{\rho}_{\mathrm{MS}}$ when $\alpha_{1} \leqslant d \leqslant \alpha_{2}$. In this case, however, the variable $t_{1}$ in equations (51), (52) cannot reach the value 1 because that would imply $S>1$ in (49). Since the largest possible value of $S$ is 1 , the optimum value of $t_{1}$ (and of $s_{1}$ ) is the one making $S=1$ in (49). This, in turn, implies that $p=0$. In this case the minimum- $\sigma^{2}$ density matrix is

$$
\hat{\rho}_{\mathrm{MS}}=\left(\frac{\alpha_{2}-d}{\alpha_{2}-\alpha_{1}}\right)|2\rangle\left\langle 2\left|+\left(\frac{d-\alpha_{1}}{\alpha_{2}-\alpha_{1}}\right)\right| 3\right\rangle\langle 3| .
$$



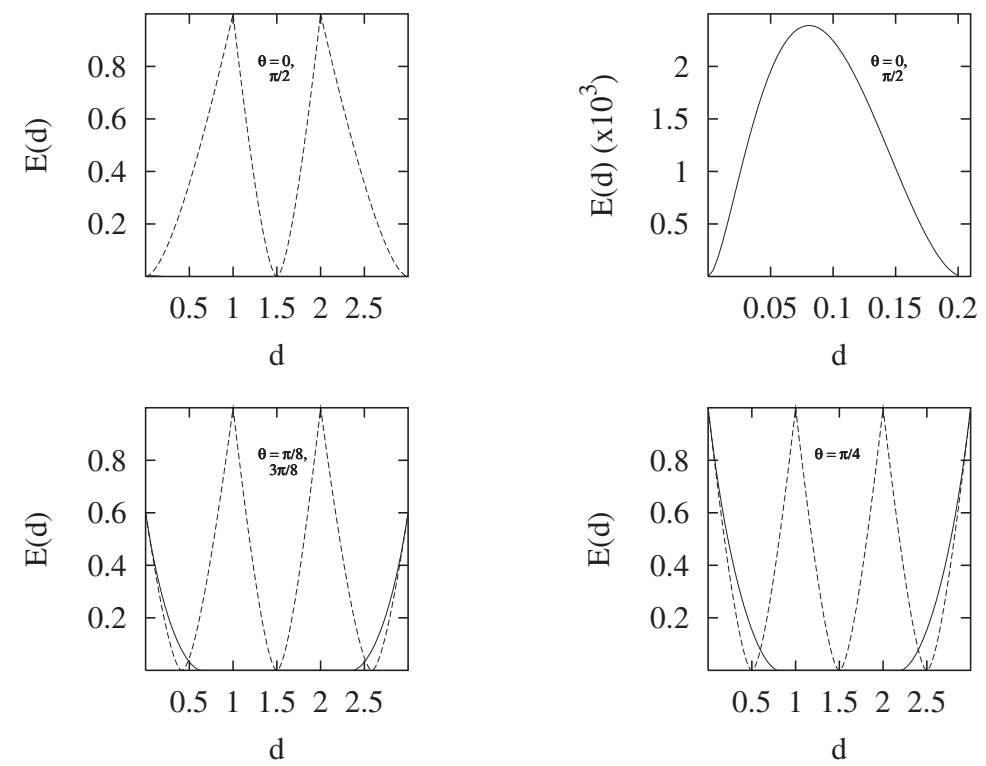

Figure 5. The entanglement of formation as a function of the expectation value of the observable $D$ with $\alpha_{1}=2$ and $\alpha_{2}=3$ (see equations (40)-(42)) evaluated, for different values of $\theta$, on (i) the MaxEnt density matrix $\exp (-\beta \hat{D}) / \operatorname{Tr}(\exp (-\beta \hat{D})$ (solid curves) and (ii) the state exhibiting the minimum value of $\left\langle\hat{D}^{2}\right\rangle$ compatible with $\langle\hat{D}\rangle$ (dashed curves).

An interesting feature of the minimum- $\sigma^{2}$ density matrix associated with $\hat{D}$ is that, for this observable, the requirement of minimizing $\left\langle\hat{D}^{2}\right\rangle$ under the constraint imposed by $\langle\hat{D}\rangle$ completely determines the matrix $\hat{\rho}_{\text {MS }}$. That is, the MaxEnt principle plays no role whatsoever when implementing Rajagopal's prescription for the observable $\hat{D}$. This seems to be a consequence of the non-degenerate character of the eigenvalues of $\hat{D}$. The entanglement of formation $E\left(\hat{\rho}_{\mathrm{MS}}\right)$ of the minimum- $\sigma^{2}$ state, as a function of the input data $d=\langle\hat{D}\rangle$, is compared in figure 5 , for different values of $\theta$, with the entanglement of formation $E\left(\hat{\rho}_{\mathrm{ME}}\right)$ of the standard MaxEnt state

$$
\hat{\rho}_{\mathrm{ME}}=\frac{1}{Z} \exp (-\beta \hat{D})
$$

where $Z=\operatorname{Tr}(\exp (-\beta \hat{D}))$. The most remarkable feature of figure 5 is that, for extended ranges of $d$-values, the minimum- $\sigma^{2}$ state is much more entangled than the standard MaxEnt state. Hence, in this case $\hat{\rho}_{\mathrm{MS}}$ is likely to create a larger amount of fake inferred entanglement than the one generated by $\hat{\rho}_{\mathrm{ME}}$. As a matter of fact, those values of $d$ leading to a separable MaxEnt matrix $\hat{\rho}_{\mathrm{ME}}$ and to an entangled matrix $\hat{\rho}_{\mathrm{MS}}$ provide explicit examples of fake entanglement generated by Rajagopal's scheme, the standard MaxEnt matrix itself describing the separable state compatible with the input information. It is remarkable that this occurs even in the case $\theta=\pi / 4$, corresponding to input data associated with an observable diagonal in the Bell basis.

Finally, notice that the study we have done in this section can be extended to the general case where the input information consists of the expectation value of an arbitrary observable endowed with a non-degenerate spectrum. Given an observable

$$
\tilde{D}=\sum_{i=1}^{4} d_{i}|i\rangle\langle i|
$$


with $d_{1}<d_{2}<d_{3}<d_{4}$, let us consider the new observable

$$
\hat{D}=\frac{\tilde{D}-d_{1} \hat{I}}{d_{2}-d_{1}}
$$

It is clear that the operator $\hat{D}$ is of the form (40), with $\alpha_{1}=\left(d_{3}-d_{1}\right) /\left(d_{2}-d_{1}\right)$ and $\alpha_{2}=\left(d_{4}-d_{1}\right) /\left(d_{2}-d_{1}\right)$, and that the minimization of $\left\langle\hat{D}^{2}\right\rangle$ for a given value of $\langle\hat{D}\rangle$ is equivalent to the minimization of $\left\langle\tilde{D}^{2}\right\rangle$ for a given value of $\langle\tilde{D}\rangle$.

\section{Conclusions}

As shown by Horodecki et al [1], the quantum state obtained by recourse to the standard MaxEnt inference prescription may be an entangled one even if there exist separable states compatible with the prior data. This situation constitutes a particularly clear instance of the problem of 'fake' inferred entanglement. In order to overcome this difficulty, but still within the strictures of the standard MaxEnt perspective, Rajagopal advanced an alternative approach to the inference of entangled states [4]. His idea is that of considering the MaxEnt state consistent with both the mean value of the observable $\hat{A}$ one is interested in and the mean value of its square $\hat{A}^{2}$, adopting for $\left\langle\hat{A}^{2}\right\rangle$ the minimum value compatible with a given value of $\langle\hat{A}\rangle$. In the case of Horodecki's example (where the prior information consists of the expectation value of the Bell operator) Rajagopal's procedure yields a separable state whenever there are separable states compatible with the available data [4]. This, together with other results recently reported in the literature $[3,5]$, constituted evidence supporting the idea that the minimum- $\sigma^{2}$ scheme may provide an appropriate and general way of solving the problem of fake inferred entanglement. However, all the aforementioned evidence was based on the study of particular examples in which the prior information was related to the Bell-CHSH observable (diagonal in the Bell basis). In order to find out to what extent the minimum- $\sigma^{2}$ prescription provides a reliable inference scheme of general applicability, we have explored here its performance when the prior information is related to more general observables, emphasizing those situations involving observables non-diagonal in the Bell basis. We have found explicit examples, related to this kind of observable, in which the minimum- $\sigma^{2}$ inference procedure leads to entangled density matrices even if there exist separable states compatible with the input data. This means that the minimum- $\sigma^{2}$ prescription is not free from the fake entanglement difficulty.

There is no doubt that Jaynes' MaxEnt principle has an important role to play in any appropriate scheme for the inference of entangled quantum states. Indeed, one of the most remarkable features of Jaynes' principle is its robustness: usually, when it seems to fail, the real problem is not the inadequacy of the MaxEnt principle itself, but rather that some piece of relevant (prior) information is not being taken into account. As recently pointed out by Brun et al [7], the various inference schemes recently advanced to solve the fake inferred entanglement problem admit of an interpretation within the strictures of Jaynes' approach. These inference prescriptions may be regarded as implementations of the MaxEnt principle in which some extra prior information (that may not consist just of the expectation values of some observables) is assumed to be known. This is certainly the case with Rajagopal's MaxEnt minimum- $\sigma^{2}$ proposal, which assumes extra information related to the square of the relevant observable. However, the results reported here show that this approach works only in very special situations.

Besides enabling us to asses the usefulness of the minimum- $\sigma^{2}$ scheme, the present effort also sheds some new light on the entanglement features exhibited by the standard 
MaxEnt principle within contexts more general than those previously considered in the literature [1,3-5].

\section{Acknowledgments}

This work was partially supported by the AECI Scientific Cooperation Program, by the DGES grants PB98-0124 and SB97-26373862 (Spain), and by CONICET (Argentine Agency).

\section{References}

[1] Horodecki R, Horodecki M and Horodecki P 1999 Phys. Rev. A 591799

[2] Buzek V, Drobny G, Adam G, Derka R and Knight P L 1997 J. Mod. Opt. 441607

[3] Rigo A, Plastino A R, Plastino A and Casas M 2000 Phys. Lett. A 2701

[4] Rajagopal A K 1999 Phys. Rev. A 604338

[5] Abe S and Rajagopal A K 1999 Phys. Rev. A 603461

[6] Sancho J M G and Huelga S F 2000 Phys. Rev. A 611

[7] Brun T A, Caves C M and Schack R 2001 Phys. Rev. A 63042309 (Brun T A, Caves C M and Schack R 2000 Preprint quant-ph/0010038)

[8] Hoi-Kwong Lo, Popescu S and Spiller T (ed) 1998 Introduction to Quantum Computation and Information (Singapore: World Scientific)

[9] Williams C P and Clearwater S H 1997 Explorations in Quantum Computing (Berlin: Springer)

[10] Williams C P (ed) 1998 Quantum Computing and Quantum Communications (Berlin: Springer)

[11] Ekert A 1991 Phys. Rev. Lett. 67661

[12] Bennett C H, Brassard G, Crepeau C, Jozsa R, Peres A and Wootters W K 1993 Phys. Rev. Lett. 701895

[13] Bennett C H and Wiesner S J 1993 Phys. Rev. Lett. 692881

[14] Ekert A and Jozsa R 1996 Rev. Mod. Phys. 68733

[15] Berman G P, Doolen G D, Mainieri R and Tsifrinovich V I 1998 Introduction to Quantum Computers (Singapore: World Scientific)

[16] Peres A 1993 Quantum Theory: Concepts and Methods (Dordrecht: Kluwer)

[17] Balian R 1991 From Microphysics to Macrophysics (Berlin: Springer)

[18] Buzek V, Adam G and Drobny G 1996 Ann. Phys., NY 24536

[19] Clauser J F, Horne M A, Shimony A and Holt R A 1969 Phys. Rev. Lett. 23880

[20] Bennett C H, DiVicenzo D P, Smolin J and Wootters W K 1996 Phys. Rev. A 543824

[21] Wootters W K 1998 Phys. Rev. Lett. 802245

[22] Peres A 1996 Phys. Rev. Lett. 771413

[23] Horodecki M, Horodecki P and Horodecki R 1996 Phys. Lett. A 2231 